\title{
Empresas transnacionales y derechos
}

humanos: La tortuosa búsqueda de responsabilidades en una globalización injusta e insostenible

Transnational Corporations and Human Rights: The Tortuous Search for Responsibility in an Unfair and Unsustainable Globalization

Autor: José Elías Esteve Moltó 


\section{EMPRESAS TRANSNACIONALES Y DERECHOS HUMANOS: LA TORTUOSA BÚSQUEDA DE RESPONSABILIDADES EN UNA GLOBALIZACIÓN INJUSTA E INSOSTENIBLE}

Transnational Corporations and Human Rights: The Tortuous Search for Responsibility in an Unfair and Unsustainable Globalization

\section{José Elías Esteve Moltó}

Profesor Titular de Derecho Internacional Público y Relaciones Internacionales Director del Máster de Derechos Humanos, Democracia y Justicia Internacional Universitat de València, España

j.elias.esteve@uv.es

Recepción: Noviembre 26 de 2020

Aceptación: Abril 19 de 2021

\section{RESUMEN}

El presente artículo tiene como objeto examinar de una manera crítica las evidentes lagunas que presenta el derecho internacional en materia de exigencia de responsabilidades a las corporaciones transnacionales por la comisión de graves violaciones a los derechos humanos. Ante el gran reto que presenta la sistemática impunidad de estas personas jurídicas, la única respuesta puede ser la regulación de sus actividades. Siendo así, por un lado, este breve análisis plantea que la exigencia de responsabilidades a las empresas debe provenir tanto del orden internacional (y no sólo a través de mecanismos propios del softlaw como los Principios Rectores de la ONU, sino mediante normas vinculantes derivadas de un tratado internacional), como del interno, a través de leyes nacionales. Y junto a esa nueva normativa que va emergiendo en algunos Estados, se requiere de un ejercicio judicial audaz tanto por parte de los

tribunales nacionales, como por un Tribunal Penal Internacional cuyo Estatuto merecería ser reformado.

\section{PALABRAS CLAVES}

Empresas transnacionales; responsabilidad corporativa; principios rectores; Planes de Acción nacional; Grupo de Trabajo sobre Empresas y Derechos Humanos.

\section{ABSTRACT}

The purpose of this article is to critically examine the obvious gaps in international law in terms of holding transnational corporations accountable for the commission of serious human rights violations. Faced with the great challenge presented by the systematic impunity of these legal entities, the only answer may be to regulate their activities. This being the case, on the one

* Artículo de reflexión 
hand, this brief analysis proposes that the requirement of responsibilities to companies must come both from the international order (and not only through soft-law mechanisms such as the UN Guiding Principles, but through binding norms derived from an international treaty), as well as from the internal one, through national laws. And together with this new regulation that is emerging in some States, a bold judicial exercise is required both by national courts and by an International Criminal Court whose Statute deserves to be reformed.

\section{KEYWORDS}

Transnational companies; corporate responsability; guiding principles; National Action Plans; Working Group on Business and Human Rights.

\section{INTRODUCCIÓN}

Desde hace décadas, organizaciones internacionales como la ONU o la OCDE han lanzado varias iniciativas para tratar de ajustar los negocios de las empresas transnacionales a unos parámetros aceptables en materia de derechos humanos. Con todo, la realidad muestra que la tarea de regular las actuaciones de las personas jurídicas se presenta impostergable y compleja, ya que de forma inherente se debe reformar un sistema comercial global, injusto y desleal. De entre los diversos instrumentos destacan los Principios Rectores sobre las empresas y los derechos humanos: puesta en práctica del marco de las Naciones Unidas para "proteger, respetar y remediar"; iniciativa que ha sido de forma generalizada apoyada por los Estados y las empresas, y criticada por las algunas organizaciones de derechos humanos. Estos principios se han tratado de llevar a la práctica a través del Grupo de Trabajo sobre Empresas y Derechos Humanos de la ONU y de los llamados Planes de Acción Nacional. Con todo, estos mecanismos autorregulatorios se vislumbran como insuficientes, de ahí que a nivel internacional se esté negociando un tratado sobre la materia; y, a nivel nacional, algunos países están empezando a legislar la cuestión empresarial. Se trata pues de exigir responsabilidades penales y civiles a nivel nacional e internacional a estos actores no estatales, cuyos privilegios y derechos no se corresponden con sus más que exiguas obligaciones.

\section{El estado de la cuestión: las empresas transnacionales como ganadoras de una injusta e insostenible globalización}

Desde hace décadas el crecimiento económico desmesurado de las empresas transnacionales -cuyos ingresos han rebasado el PIB de numerosos Estados ${ }^{1}-$ y sus acreditados y reconocidos efectos perversos sobre los derechos humanos y el medio ambiente ${ }^{2}$ han escapado al control del derecho internacional y de las legislaciones nacionales. El comportamiento de estos actores no estatales, que ha sido calificado de psicótico (Bakan, 2004), les ha generado el título de ser los grandes ganadores de la globalización económica y comercial (Stiglitz, 2007, pp. 3-60). Pero no únicamente han amasado un gran poder económico a cualquier precio, sino que sus interferencias en los asuntos públicos han sido notorias y relevantes hasta llegar a variar el destino de los pueblos. Baste recordar los golpes de Estado, entre otros, contra los gobiernos de

1. Entre otras pueden consultarse las estadísticas comparativas en distintas fuentes bibliográficas, donde por reseñar algunos ejemplos, los ingresos de la General Motors superan el PIB conjunto de Dinamarca y Tailandia o los de la empresa japonesa Toyota que rebasan el PIB aunado de Portugal y Malasia (ver, Wells, C. y Elias, J.).

2. Numerosas obras interdisciplinares, hacen referencia al actual sistema que favorece el enriquecimiento a cualquier precio de estas empresas, entre las que se pueden citar, Hernández Zubizarreta, J.: Las empresas transnacionales frente a los derechos humanos. Historia de una asimetría normativa. Ed Egoa, Vitoria. 2009. Teiltelbaum, A.: Al margen de la ley: sociedades transnacionales $y$ derechos humanos, Corporación colectivo de abogados José Alvear Restrepo, ILSA, Observatorio Social de Empresas Transnacionales, Bogotá, 2007. Ziegler, Jean: El poder de la vergüenza, Editorial Taurus, 2006. Stiglitz, J.: La Guerra de los tres billones de dólares, Taurus, Madrid, 2008. Napoleoni, Loretta: La economía canalla, Editorial Paidos, 2008. Artus, P. y Virard, M. P.: Globalizacion. Lo peor está por llegar, editorial Icaria, 2009. Klein, Naomi: La doctrina del shock, ed. Paidós, 2010 y de la misma autora, No logo. El poder de las marcas, ed. Paidós, 2007. George, Susan: Sus crisis, nuestras soluciones. ed. Icaria, Intermón Oxfam, 2010. Kingsnorth, Paul: Un no, muchos síes, Ediciones del Bronce, Planeta, 2004. 
Mossadegh en Irán (1953), de Jacobo Arbenz en Guatemala (1954) y Salvador Allende en Chile (1973) que no hubieran sido posibles sin la complicidad respectivamente de empresas transnacionales, tales como la Anglo-Iranian Oil Company, la United Fruit Company y la International Telephone and Telegraph que se opusieron en dichos países a la nacionalización del petróleo, a la reforma agraria y a la gestión pública del servicio de telefonía ${ }^{3}$.

Ahora bien, el fenómeno de la proliferación de estos agentes económicos se ha desarrollado de forma exponencial desde la progresiva retirada de los aranceles comerciales que ha provocado la masiva deslocalización empresarial. En efecto se ha ido trasladando la producción de los países occidentales a los más desfavorecidos a través de distintas fórmulas jurídicas, como las sucursales, filiales, por ejemplo. Gracias a estos intrincados ejercicios de ingeniería jurídica, las empresas matrices han sido capaces de retener el beneficio económico, mientras han deslocalizado las responsabilidades a países terceros. $\mathrm{Y}$ con ello, han podido además externalizar la polución ambiental (Lutz, 1988, pp. 629-677) y la precariedad laboral, en ocasiones evadiendo las leyes y en otras aprovechándose del limbo legal internacional en la materia.

A título ilustrativo, han sido víctimas y testigos directos de esta impunidad en relevantes procesos judiciales, los supervivientes de la India por la catástrofe industrial de la Union Carbide en Bhopal (Baxi, 2010, pp. 23-44, p. 39), los indígenas de la Amazonía de Ecuador, perjudicados por los indiscriminados vertidos de petróleo de la Chevron-Texaco (Pigrau, 2014, pp. 1-43), (Kimerling, 2006, pp. 445 y ss.), (Kenney, 2009 , pp. 857 y ss.), o el oprimido pueblo Ogoni en el Delta del Níger por el mismo tipo de vertidos de la Shell en Nigeria. Como

3. Véase la sustanciosa monografía del representante de la Asociación Americana de Juristas en Ginebra, Teitelbaum, A.: El papel de las sociedades transnacionales en el mundo contemporáneo. Documento de trabajo para el seminario de sociedades transnacionales, Ed. CETIM/ AAJ, Ginebra, mayo 2001, pp.10-13. es bien sabido, este último asunto ha dado lugar a uno de los litigios más relevantes, que ha venido a cuestionar el avance judicial de las víctimas ante los tribunales norteamericanos, amparando la más manifiesta ausencia de responsabilidades de las empresas transnacionales, que de nuevo quedó palmariamente manifestada en la controvertida sentencia de su Tribunal Supremo en el asunto Kiobel (Zamora, 2016, pp. 149-166), (Marullo \& Zamora, 2016), (Grear \& Weston, 2015, pp. 21-44).

En la actualidad al imparable avance corporativo se van sumando otras desafiantes realidades para el derecho internacional. El desenfrenado fenómeno del acaparamiento de tierras (Zamora, 2013), que recorre distintas regiones ricas en recursos y que se asientan en los enclaves donde la población queda más desprotegida, como en África (Nseeuw, 2013, pp. 159-177). El saqueo de recursos, al que se han unido el voraz apetito de las empresas de la nueva potencia emergente, China (Zolin y Braggion, s.f.). Grandes avances se deben realizar en un breve lapso para que los tan cacareados Objetivos para el supuesto Desarrollo Sostenible no sean otro papel mojado para las Naciones Unidas. Y es que estos principios no pasarán de la retórica mientras no se actúe para tratar de atajar (entre otros muchos frentes), los perniciosos efectos de la cadena de suministros de estos conglomerados empresariales, que se vislumbran, por ejemplo, en los monocultivos de algodón en India o Uzbekistán o de la palma para la extracción de aceite en Malasia o Indonesia.

Asimismo, urge poner un mínimo control en el negocio de las semillas transgénicas y sus respectivos pesticidas, cuyas amenazas parecen agrandarse tras la adquisición de Monsanto por la alemana Bayer. Igualmente, impensables progresos legales se deben dar para evitar la impunidad de las empresas transnacionales (Zamora, 2012, pp. 194-207), como Trafigura (registrada en Londres) la cual fletó un buque de pabellón panameño, el Probo Koala, para depositar residuos tóxicos en las playas de la capital de Costa de Marfil, Abiyán, diezmando a parte de la población, y cuyos efectos han sido calificados de crimen 
contra la humanidad (Manirabona, 2011, pp. 535-576); o cómo limitar y sancionar con eficacia el extractivismo rampante y contaminante de las transnacionales mineras con independencia del país donde se encuentren registradas, ya se trate de empresas privadas como las canadienses o incluso con participación pública como las chinas, las cuales empiezan a asentarse en países latinoamericanos para explotar los recursos de territorios indígenas, ya sean los del pueblo Shuar -en la cordillera del Cóndor en Ecuador- o los que habitan distintas regiones nicaragüenses y cuyas tierras están siendo expropiadas para construir el megaproyecto de canal que pretende unir por segunda vez- los dos grandes océanos a través del lago Nicaragua.

La tarea de regular las actuaciones de las empresas transnacionales se presenta titánica, ya que de forma inherente se debe reformar un sistema comercial injusto y desleal (Moon, 2013, pp. 1-41). Se trata pues de exigir responsabilidades penales y civiles a nivel nacional e internacional a estos actores no estatales, cuyos privilegios y derechos no se corresponden con sus nulas obligaciones. Resulta impostergable poner límites, por ejemplo a una industria textil que en Bangladesh provocó el derrumbe del edificio Rana Plaza en el que murieron 1.135 personas $^{4}$ o que más recientemente en Myanmar han comenzado a explotar a su paupérrima población desprotegida en las llamadas Zonas Económicas Especiales ${ }^{5}$, y que durante un largo periodo se han lucrado, gracias al mantenimiento en el poder de dirigentes corruptos y dictatoriales (Dale, 2011), (Burna, 2008), que no han dudado

4. Tragedia criminal que trata de exigir responsabilidades alguno de los mandos de la empresa ("Acusan a 38 personas de asesinato por el derrumbe del edificio Rana Plaza en Bangladesh", eldiario.es, 19 julio 2016 en http://www.eldiario.es/desalambre/Acusan-asesinatoRana-Plaza-Bangladesh_0_538946139.html), pero que en todo caso la legislación nacional y la laguna jurídica internacional en la materia, siempre acaban avalando la impunidad de las empresas matrices localizadas en países occidentales, cfr. S. Labowitz y D. Baumann-Pauly: Beyond the tip of the iceberg: Bangladesh's forgotten apparel workers, Nyu Stern, Center for Business and Human Rights, New York, diciembre 2015.

5. International Commission of Jurists: Special economic zones in Myanmar and the State duty to protect human rights, ICJ, Ginebra, 2017. en cometer un genocidio contra la minoría Rohingya, al sur del país.

\section{Las Naciones Unidas y sus planes autorregulatorios, ante la creciente impunidad de las corporaciones transnacionales}

Ante el desafío corporativo mencionado, desde la década de los 70 han proliferado las iniciativas de Naciones Unidas y otras privadas, recomendando la fijación de límites a las empresas. Una de estas primeras propuestas vino impulsada por el Consejo Económico y Social por medio de la creación, tanto de la Comisión de Sociedades Transnacionales como del Centro de Sociedades Transnacionales. A estos organismos se les encomendó la misión de elaborar un código de conducta para estos actores no estatales. Ahora bien, la ausencia de consenso respecto a la primera versión del proyecto del código de conducta de la Comisión de Sociedades Transnacionales ${ }^{6}$ (Ver Fikentscher, 1982, pp. 577-604), al margen de impedir su aprobación, por la imposibilidad de reconciliar las posturas enfrentadas respecto a su carácter vinculante, acabó por provocar en 1993 la transformación de este organismo en una oficina subordinada a la Conferencia de las Naciones Unidas sobre el Comercio y Desarrollo.

Pocos años después el entonces Secretario General de las Naciones Unidas, Kofi Annan, lanzaba su propia iniciativa el 31 de enero de 1999 presentando en el Foro Económico Mundial en Davos el llamado Global Compact (Pacto Global). Esta plataforma de las Naciones Unidas ha sido justamente criticada, en primer lugar, por agrupar como socios, no sólo a Estados y ONG's, sino a las mismas empresas transnacionales, las cuales financian gran parte de este mecanismo. Además, su contenido, agrupado en diez principios genéricos y difusos ${ }^{7}$, pretende

6. La primera versión del código de conducta data de 1982, E/C.10/1982/6.

7. "Los Diez Principios del Pacto Mundial están basados en Declaraciones y Convenciones Universales aplicadas 
que sea asumido de buena fe y de forma voluntaria por las empresas. A ese carácter no vinculante del acuerdo se le añade que el único mecanismo para verificar el cumplimiento de esos estándares en materia de derechos humanos, laborales y medioambientales, queda en manos de las propias empresas que se convierten en juez y parte de sus actividades. Circunstancia que no ha pasado inadvertida a la misma Dependencia Común de Inspección de las Naciones Unidas quien en su informe de evaluación del Pacto Global, sin ningún tipo de ambigüedades ha considerado que esta estrategia ha contribuido "a legitimar el compromiso de la organización con el sector privado durante años. Con todo la carencia de un mandato claro y articulado ha dado lugar a un enfoque y un impacto confusos: la ausencia de criterios de ingreso adecuados y de un sistema de vigilancia eficaz para medir la puesta en práctica real de los principios ha despertado críticas y creado un riesgo para la reputación de la Organización"8.

Ante la falta de credibilidad del Pacto Global, en julio de 2005 el Secretario General siguiendo las instrucciones de la Comisión de Derechos Humanos designó a John Ruggie como Representante Especial para los Derechos Humanos y las empresas transnacionales con un mandato preciso. Tras seis años de investigación y redacción de propuestas, finalmente el 6 de julio de 2011 el Consejo de Derechos Humanos de las Naciones Unidas aprobaba una serie de principios $^{9}$ que pretendían ordenar los efectos de las actuaciones de las empresas transnacionales sobre los derechos humanos. Estas directrices han llegado a calificarse por

en cuatro áreas: Derechos Humanos, Medio Ambiente Estándares Laborales y Anticorrupción. en http://www. unglobalcompact.org/Languages/spanish/Los_Diez_ Principios.html.

8. Informe de la Dependencia Común de Inspección de las Naciones Unidas, United Nations corporate partnerships. The role and functioning of the Global Compact, JIU/ REP/2010/9. El documento concluye apuntando que: "Diez años después de su creación, a pesar de la actividad intensa realizada por el Global Compact y de los recursos cada vez mayores que ha recibido, los resultados son ambiguos y los riesgos no han disminuido".

9. Resolución del Consejo de Derechos Humanos de 6 de julio de 2011, A/HRC/RES/17/4, 17º período de sesiones. la misma organización como "un paso sin precedentes", ya que se argumenta que ha creado un marco global con el propósito de prevenir y afrontar los negativos impactos derivados de la actividad económica de estos nuevos y poderosos agentes de la globalización ${ }^{10}$.

Así pues, con esta resolución, el Consejo hizo suyo el informe del Representante Especial del Secretario General que contiene los "Principios Rectores sobre las empresas y los derechos humanos: puesta en práctica del marco de las Naciones Unidas para "proteger, respetar y remediar"'"11, considerando que esta estrategia ofrece una nueva orientación que contribuye al objetivo de una "globalización socialmente sostenible". Para ello se diseñaron tres grandes pilares de actuación en este nuevo Marco, como anunció Ruggie en su planteamiento en el informe del 2008, los cuales se desglosan, en primer lugar, en un deber de los estados de proteger los derechos humanos, en segundo lugar se dispone de la responsabilidad de las empresas de respeto de los mismos, para finalizar en tercer lugar, con la reparación de los daños causados por la trasgresión de tales derechos.

Ahora bien más allá del pulcro ejercicio retórico de la iniciativa, su eficacia queda cuestionada desde sus inicios. El punto 14 de la misma Introducción ya viene a precisar que los Principios Rectores no entrañan una aportación normativa de la que se desprenda la asunción de nuevas obligaciones en materia de derechos humanos ${ }^{12}$; advertencia

10. UN Press release OHCHR (Office of the High Commissioner for Human Rights): New guiding principles on business and Human Rights endorsed by the UN Human Rights Council en http://www. ohchr.org/en/NewsEvents / Pages/DisplayNews. aspx? NewsID=11164\&LangID=E.

11. Informe del Representante Especial del Secretario General para la cuestión de los derechos humanos y las empresas transnacionales y otras empresas, John Ruggie. Principios Rectores sobre las empresas y los derechos humanos: puesta en práctica del marco de las Naciones Unidas para "proteger, respetar y remediar". A/ HRC/17/31 de 21 de marzo de 2011.

12. "La aportación normativa de los Principios Rectores no radica en la creación de nuevas obligaciones de derecho internacional, sino en precisar las implicaciones de las normas y métodos actuales para los Estados y las empresas; en integrarlas en un modelo único lógicamente 
que se ratifica en los párrafos preliminares del anexo que contiene los principios y que declara que: "En ningún caso debe interpretarse que estos Principios Rectores establezcan nuevas obligaciones de derecho internacional ni que restrinjan o reduzcan las obligaciones legales que un Estado haya asumido, o a las que esté sujeto de conformidad con las normas de derecho internacional en materia de derechos humanos"13.

Asimismo, se preocupa el Informe de establecer de forma extensa la "debida diligencia" de las empresas en la prevención, mitigación y reparación de las infracciones de los derechos humanos. A este fin deberían llevar a cabo las siguientes actuaciones: "evaluación del impacto real y potencial de las actividades sobre los derechos humanos, la integración de las conclusiones, y la actuación al respecto; el seguimiento de las respuestas y la comunicación de la forma en que se hace frente a las consecuencias negativas"14. Lo llamativo de estos procesos internos de prevención, reside en el hecho que en ningún momento se hace mención sobre la posibilidad de establecer mecanismos externos por la propia ONU para verificar si esa debida diligencia es un hecho o una quimera. Únicamente se hace referencia a la voluntaria potestad de las empresas para someterse a indicadores cualitativos y cuantitativos, inspecciones y auditorías; claro está sin precisarse quién debe proceder a efectuar esas evaluaciones, dejando así todo la "diligencia debida" en la misma e hipotética empresa infractora o en una consultora contratada por ella misma a título individual. Como colofón de la responsabilidad de respetar los derechos humanos por parte de las empresas, el informe recomienda un principio operativo de dudosa reparación, ya que ésta únicamente puede surgir si las empresas determinan ellas mismas que han violado

coherente e inclusivo; y en reconocer los puntos débiles del actual sistema y las mejoras posibles.", A/HRC/17/31, punto 14 de la Introducción de los Principios Rectores.

13. A/HRC/17/31, op. cit. p. 7.

14. A/HRC/17/31, op. cit. p. 18. los derechos humanos, y que además dicho quebrantamiento ha causado un daño.

Habida cuenta no sólo del carácter voluntario de los Principios, sino de su peculiar sistema de autorregulación, no resulta extraño que fundamentadas críticas hayan sembrado dudas sobre el proyecto, ya que "voluntary measures are typically unevenly applied, not sufficiently independent, transparent and unbiased, may be used strategically to thwart agency by communities struggling to protect values of importance to them, and cannot compel sanction or remedy"15.

Ahora bien, una vez enunciados los Principios, debían los mismos hacerse efectivos, y para ello el Consejo de Derechos Humanos impulsó la creación de un Grupo de Trabajo sobre Empresas y Derechos Humanos de la ONU. Precisamente los expertos independientes de este mecanismo extraconvencional no han dejado de insistir desde sus inicios que los Estados deben aplicar a nivel interno los Principios a través de los llamados Planes de Acción Nacional (PAN). Para facilitar esta tarea, en noviembre del 2016 el Grupo de Trabajo publicaba una Guía Práctica (UN Working Group on Business and Human Rights, 2016) en el que de forma sucinta describía en cinco fases el proceso que debían seguir los Estados para implementar los Principios Rectores, al mismo tiempo que remarcaba los principios

15. Having the Ruggie Pulled Out From Under Us: From "Sanction and Remedy" to non-judicial grievance mechanisms "Non-Judicial Grievance Mechanisms" en http://www.miningwatch.ca/article/having-ruggiepulled-out-under-us-sanction-and-remedy-non-judicialgrievance-mechanisms. Opinión de la organización Mining Watch Canada, en la que en su informe sobre los Principios Rectores proporciona ejemplos reales de la ineficiencia de estos mecanismos de control: "We have two non-judicial grievance mechanisms in Canada that are relevant to the activities of Canadian extractive companies operating overseas. The National Contact Point for the OECD Guidelines on Multinational Enterprises and the Canadian CSR Counsellor for the extractive sector, created in 2009 under the Government of Canada's new CSR Policy for the extractive sector called "Building the Canadian Advantage." Neither will investigate complaints; neither will make determinations of fact about whether the guidelines they are meant to uphold were actually breached, neither will provide sanction and both can only provide remedy if the corporation against which a complaint has been made decides to provide some form of remedy". 
y medidas que debían ejecutarse para dar cabal efecto a los mismos.

Con todo, esta política de promoción de PAN's curiosamente no surgió de este mecanismo de la ONU, sino de la Unión Europea (Márquez, 2017, pp. 21-47). Pocos meses después de la aprobación de los Principios de Ruggie, la Comisión en su Estrategia renovada de la UE para 20112014 sobre la responsabilidad social de las empresas instaba a los Estados a llevar a cabo sus propios planes nacionales ${ }^{16}$. Y del mismo modo el Consejo de la UE ha hecho lo propio en las distintas ediciones de su Marco Estratégico y Plan de Acción sobre Derechos Humanos y Democracia ${ }^{17}$. Por otra parte, las organizaciones regionales con competencias en derechos humanos también se han hecho eco de los llamamientos del Grupo de Trabajo de la ONU. Asimismo, en suelo europeo, el Comité de Ministros del Consejo de Europa, tras una primera Declaración de 2013 alentando a los Estados parte a adoptar los PAN, emitió en marzo de 2016 una Recomendación sobre Derechos Humanos y Empresas $^{18}$. De manera similar, la Asamblea General de la OEA ha hecho lo propio y en dos resoluciones de 2014 y 2016, tras recordar los compromisos derivados de la Agenda 2030 para el Desarrollo Sostenible ${ }^{19}$ y de los Principios Rectores, ha interpelado a los Estados miembros a ir desarrollando esos planes nacionales ${ }^{20}$. Es más, la última

16. COM/2011/681/FINAL, Estrategia renovada de la UE para 2011-2014 sobre la responsabilidad social de las empresas, 25 de octubre de 2011.

17. Consejo de la Unión Europea 11855/12, Marco Estratégico y Plan de Acción 2012-2014 sobre Derechos Humanos y Democracia de la UE de 25 de junio de 2012; Plan de Acción para los derechos humanos y la democracia (2015-2019) 10897/15, «Mantener los derechos humanos entre las prioridades de la agenda de la UE» en http://data.consilium.europa.eu/doc/ document/ST-10897-2015-INIT/es/pdf. Véanse las tres medidas del punto 18, "avanzar en lo relativo al comercio y los derechos humanos" y el punto 25 "política de comercio e inversión".

18. Recommendation $\mathrm{CM} / \operatorname{Rec}(2016) 3$ of the Committee of Ministers to member States on human rights and business (Adopted by the Committee of Ministers on 2 March 2016 at the 1249th meeting of the Ministers' Deputies).

19. Sobre esta cuestión, véase el estudio de Díaz Barrado. 20. AG/RES. 2840 (XLIV-O/14) Promoción y Protección de los Derechos Humanos en el ámbito empresarial (Aprobada en la segunda sesión plenaria celebrada el 4 resolución efectúa un encargo a la misma Comisión Interamericana de Derechos Humanos para que apoye el desarrollo de los planes y efectúe "un estudio sobre los estándares interamericanos en materia de empresas y derechos humanos" ${ }^{21}$. En esta misma línea, la Unión Africana está dedicando esfuerzos a impulsar los Principios Rectores, pero en este caso con el apoyo de la UE, y en marzo de 2017 acabó por confeccionar un borrador de Proyecto de la Unión Africana de una Política Marco sobre Empresas y Derechos Humanos. Como elemento principal de este plan se apunta a la vital importancia geoestratégica de los países africanos por su riqueza en recursos minerales y la urgente necesidad de frenar el alarmante fenómeno del acaparamiento de tierras (Adeola, R., 2017). Incluso en la región del Sudeste Asiático hasta la irrelevante y cuestionada Comisión de Derechos Humanos de la ASEAN ha dedicado alguna de sus sesiones a esta cuestión (ASEAN, 2017).

En todo caso, más allá del impulso y publicación de distintos PAN's, la pregunta que debe plantearse es si estos nuevos instrumentos derivados de los Principios de Ruggie pueden abrir cauces procedimentales a las víctimas para poder exigir responsabilidades (penales y civiles) a las empresas.

Finalmente, a los mencionados proyectos de la ONU deben añadirse con similares efectos, las voluntarias Directrices de la Organización para la Cooperación Económica y el Desarrollo (OCDE) para empresas multinacionales (OCDE, 2013) o a los mecanismos de la Declaración Tripartita de Principios sobre las empresas multinacionales

\footnotetext{
de junio de 2014); AG/RES. 2887 (XLVI-O/16) Promoción y Protección de Derechos Humanos (Aprobada en la segunda sesión plenaria, celebrada el 14 de junio de 2016).

21. "4. Solicitar a la CIDH que para el último semestre de 2016 realice un estudio sobre los estándares interamericanos en materia de empresas y derechos humanos a partir de un análisis de las convenciones, jurisprudencia e informes emanados del sistema interamericano, lo que podrá servir de insumo para los esfuerzos realizados por los Estados Miembros en varias iniciativas nacionales e internacionales en la esfera de empresas y derechos humanos.", en http://www.oas.org/ es/sla/ddi/docs/AG-RES_2887_XLVI-0-16.pdf.
} 
y la política social de la Organización Internacional del Trabajo (OIT) (OIT, 2001), que no regulan un procedimiento de denuncias. Más bien insisten en el establecimiento de códigos externos de conductas, que una vez más han sido tachados de albergar una "eficacia prácticamente nula y una efectividad básicamente retórica" (Diller, 2000). Finalmente desde esta misma perspectiva y con una cuestionada eficacia se ha extendido la aplicación de la debida diligencia a la cadena de suministro responsable de explotación de minerales en zonas de conflictos (Martin-Ortega, 2013, pp. 15 y ss.), en especial en la región de los Grandes Lagos (Cervera, 2016) y a la industria textil (OCDE, 2017).

\section{¿Será posible un tratado internacional que atribuya responsabilidades a las empresas transnacionales?}

Con independencia del soft law (Sánchez, 2016) referido en el epígrafe anterior, desde la ONU también se han lanzado propuestas para articular obligaciones de carácter vinculante para las empresas. En este sentido debe traerse a colación la labor desarrollada en su día por la Subcomisión sobre la prevención de la discriminación y protección de las minorías que creó a su vez en 1998 un Grupo de Trabajo ${ }^{22}$. Entre las misiones asignadas figuraban de una vez más la elaboración de un nuevo código de conducta. Sin embargo, tras distintos proyectos y deliberaciones acabó por presentarse a la rebautizada Subcomisión sobre la promoción y protección de los derechos humanos, las llamadas Normas de la ONU sobre la responsabilidad de las empresas transnacionales y otras empresas en relación a los derechos humanos ${ }^{23}$, las cuales fueron sometidas a un proceso de consultas por parte de la Oficina del Alto Comisionado, tal y como recomendó la anterior Comisión de Derechos Humanos. En este nuevo

22. Resolución de la Subcomisión sobre la prevención de la discriminación y protección de las minorías de 20 de agosto de 1998, E/CN.4/Sub.2/Res/1998/8.

23. E/CN.4/Sub.2/2003/12/Rev.2 de 26 de agosto de 2003. documento se identificaron y concretaron una serie de obligaciones a las cuales de forma imperativa debían ajustarse las empresas, como la no utilización de trabajos forzosos, la prohibición del trabajo infantil o el no recurrir al soborno para beneficiar a sus propios negocios; incluso se hace mención expresa a la práctica degenerativa de corromper candidatos a cargos públicos de cualquier tipo de proceso electoral (Jägers, 2002, pp. 124-128), (Hillemanns, 2003, pp. 1065-1080), (Wallace y Martin-Ortega, pp. 304-319). De la misma forma para evitar que las normas cayeran en una mera declaración de intenciones se contemplaron mecanismos para que las empresas incorporasen de forma efectiva esas obligaciones. Todo ello venía reforzado tanto por procedimientos de verificación independiente por parte de las Naciones Unidas del grado de cumplimiento de los deberes enunciados, como por la creación a nivel nacional de procedimientos para dar curso a las denuncias de los trabajadores por posibles violaciones de las normas (Weissbrodt y Kruger, 2003, pp. 901-922). Por todo ello, no resulta extraño, que finalizadas las consultas sobre estas Normas de la ONU por parte de la Oficina del Alto Comisionado de los Derechos Humanos, y constatadas las vehementes reacciones críticas del sector empresarial y de numerosos Estados, la Comisión de Derechos Humanos acabara por abandonar el documento remarcando su carácter de proyecto no vinculante ${ }^{24}$.

Justo una década más tarde y ante las expectativas frustradas de los Principios Rectores, algunos Estados han decidido retomar la estrategia de consensuar un instrumento internacional vinculante. En efecto un atisbo de esperanza parece haber vuelto a asomarse, con ocasión del $26^{\circ}$ período de sesiones del Consejo de Derechos Humanos. El 25 de junio de 2014, a iniciativa de Bolivia, Cuba, Ecuador, Sudáfrica y Venezuela se presentó un proyecto de resolución para la "elaboración de un instrumento internacional jurídicamente vinculante sobre las empresas

24. Resolución de la Comisión de Derechos Humanos, de 20 de abril de 2004, E/CN.4/Res/2004/116. 
transnacionales y otras empresas con respecto a los derechos humanos"25. De esta forma se ha establecido un Grupo Intergubernamental de Trabajo, que tiene como misión el tratar de negociar un tratado internacional.

Elmencionado Grupo Intergubernamental de Trabajo ya ha celebrado en octubre de 2020, su Sexta Sesión Anual en Ginebra con la participación de representantes estatales y de la sociedad civil, y las numerosas contribuciones aportadas que instan a los Estados a que el borrador final integre desde una perspectiva holística distintos enfoques en el tratamiento de esta problemática, como son: género, igualdad y raza (Patel \& Nyembe. 2017). Queda por ver el resultado de este Grupo en el que las contundentes demandas de las ONG's chocan frontalmente con la tibieza de algunos Estados, de la Unión Europea e incluso de un sector de los llamados expertos.

Sea como fuere, este instrumento internacional debiera ser la respuesta a las violaciones de los derechos humanos de las empresas a través de una doble vía: creando un mecanismo o tribunal internacional de rendición de cuentas, y al mismo tiempo, habilitando a los Estados ratificantes a implementar a nivel interno una serie de medidas que refuercen la acción internacional.

En efecto, en primer lugar, se propone a nivel internacional en el segundo borrador revisado de 6 de agosto de 2020 a un posible Comité independiente internacional de expertos de supervisión para el cumplimiento del tratado (artículo 15 del borrador revisado) y un Fondo Internacional para las víctimas (artículo 15.7 del borrador revisado). Precisamente a este Comité de supervisión se le atribuyen toda una serie de potestades que van desde la formulación de observaciones generales y recomendaciones a los Estados, hasta

25. Consejo de Derechos Humanos, 26 a período de sesiones, A/HRC/26/L22/Rev.1, 25 junio de 2014, "Elaboración de un instrumento jurídicamente vinculante sobre las empresas transnacionales y otras empresas con respecto a los derechos humanos". la presentación de un informe anual a la Asamblea General de la ONU. Y como sucede, con este tipo de mecanismos convencionales onusianos, un Protocolo Adicional se habilita para que se pueda habilitar la competencia de este hipotético Comité para poder examinar denuncias individuales o grupales de víctimas de violaciones de derechos humanos cometidas como consecuencia de una actividad empresarial. Este control internacional propuesto en este borrador de tratado se aleja claramente de las pretensiones más ambiciosas iniciales, que hacían referencia, bien a la creación de un nuevo tribunal penal internacional para empresas transnacionales, bien a la reforma del Estatuto de Roma del Tribunal Penal Internacional para poder perseguir a estas personas jurídicas.

Junto a esta exigencia de responsabilidades en el orden internacional, los Estados según indica el artículo cuarto del borrador y bajo la perspectiva del principio de complementariedad, deben garantizar a nivel interno a víctimas y familiares de dichos abusos empresariales, el acceso justo, no discriminatorio y efectivo a la justicia (artículo 4.2.c del borrador revisado). Siendo así, los Estados estarían obligados tanto a aprobar leyes nacionales para facilitar tanto el acceso a las víctimas a la justicia, como la competencia jurisdiccional de los tribunales para este tipo de casos (artículo 7.2 del borrador de tratado). Es más, se contempla la obligación de los tribunales nacionales donde radica el domicilio de la empresa que deben ejercer su jurisdicción con independencia de la nacionalidad de las víctimas, desestimando así desde un inicio el acudir al manido argumento del forum non conviniens para desestimar este tipo de casos. En este sentido, resulta relevante destacar que el borrador revisado de tratado prevé asimismo en su artículo 12 la obligación de cooperación judicial internacional y la asistencia legal mutua entre los Estados en la investigación abierta de procedimientos civiles, administrativos y penales.

En definitiva, de aprobarse dicho tratado se estarían instaurando obligaciones para las empresas y un sistema de rendición de 
cuentas y exigencia de responsabilidades a nivel internacional e interno, apuntando directamente a estos conglomerados económicos y comerciales como nuevos sujetos de derecho internacional.

\section{La vía de la exigencia de responsabilidades a través de leyes nacionales}

Al margen del proyecto de tratado internacional, otras iniciativas de carácter vinculante para las empresas se están gestando a nivel interno de los Estados. En efecto los Principios Rectores aunque desde la perspectiva internacional dejan sin resolver la posible extensión de la responsabilidad de proteger del Estado respecto a las actividades de las empresas filiales que operan fuera del territorio donde radica la empresa matriz -un pormenorizado estudio del contenido de estos informes anuales del Representante Especial se puede consultar en Weilert (2010, pp. 445-506 y pp. 491-504)-. La implementación de los mismos a través de sus planes nacionales de acción (Márquez, 2015, pp. 55-87), pudiera derivar en obligaciones internas para estos actores no estatales.

Animados por el impulso de la Eurocámara de Bruselas, a través de la Resolución del Parlamento Europeo, de 25 de octubre de 2016, sobre la responsabilidad de las empresas por violaciones graves de los derechos humanos en terceros países ${ }^{26}$, en Francia por ejemplo el 21 de febrero de 2017 la Asamblea Nacional acabó de aprobar una ley de diligencia debida que pretende controlar a las empresas matrices, aunque el Consejo Constitucional ha cuestionado alguna de sus disposiciones (Debouzy, 2017). A pesar de ello, esta ley francesa ha comenzado a desplegar sus efectos judiciales. En efecto, en el contexto de la guerra de Siria la empresa

26. Resolución del Parlamento Europeo, de 25 de octubre de 2016, sobre la responsabilidad de las empresas por violaciones graves de los derechos humanos en terceros países (2015/2315(INI)), en http://www. europarl.europa.eu/sides/getDoc.do?pubRef=-//EP// TEXT+TA+P8-TA-2016-0405+0+DOC+ XML +V0//ES. filial francesa en este país, la cementera Lafarge Cement Syria, ha sido acusada ante los tribunales de París de acciones cómplices en la comisión de crímenes de guerra y financiación del terrorismo, entre otros delitos, supuestamente cometidos en Jalabiya (noreste Siria). En este caso la justicia francesa ya ha admitido a trámite la querella y está investigando cómo la empresa Lafarge continuó en la zona ocupada por el ISIS y les compraba petróleo, y materias primas; cómo continuó trabajando en la zona, contribuyendo al pago revolucionario de tasas a los terroristas; por qué repatrió a empleados occidentales, pero obligó a empleados sirios a continuar trabajando, algunos incluso durmiendo en la cementera; y cuál es su responsabilidad respecto a los empleados sirios desaparecidos mientras iban al lugar de trabajo, en cuya ruta debían atravesar diariamente los checkpoints del ISIS (ECCHR, 2016).

Los mismos debates parlamentarios se están produciendo en los Países Bajos a propósito del trabajo infantil, y en Suiza donde se va a celebrar un referéndum sobre esta cuestión a finales de noviembre de 2020, y que afecta a las empresas transnacionales o en los países nórdicos, como Dinamarca, Finlandia y Suecia, que han aprobado ya sus respectivos planes de acción. En el Reino Unido por su parte ya ha promulgado su plan de acción nacional ${ }^{27}$, junto a la Modern Slavery $A c t^{28}$; ley pionera y cuyo marco regulador se debate asimismo en Australia. Sea como fuere, los primeros efectos de la ley británica ya han tenido eco ante sus tribunales con una primera sentencia condenatoria de enero de 2018 (Thomson Reuters Foundation, 2018).

Igualmente la novedosa creación en Canadá de la figura de un Defensor del Pueblo para la Responsabilidad de las Empresas (CORE, Canadian Ombudsperson for Responsible Enterprise) y de un órgano consultivo en el que la sociedad civil a través

27. El Reino Unido ya ha publicado su Plan Nacional; vid. en http://www.business-humanrights.org/ UNGuidingPrinciplesPortal/ToolsHub/Governments/ UKNationalActionPlan

28. Modern Slavery Act 2015 en http://www.legislation. gov.uk/ukpga/2015/30/contents/enacted. 
de esta nueva institución podrá advertir al ejecutivo $\mathrm{y}$ al nuevo Ombudsperson de violaciones a los derechos humanos cometidas por empresas canadienses en terceros países, supone un paso más, aunque un tanto tibio, para poder facilitar la reclamación de daños por parte de las víctimas (Business and Human Rights Resource Centre, 2018).

En este sentido, aunque con sus limitaciones, pudieran ser también fuente de inspiración los avances legislativos, tales como las que se han llevado a cabo en los Estados Unidos con la Dodd-Frank Financial Reform Act, Section 1502, con la que el presidente Obama pretendía dar los primeros pasos para regular las actuaciones de Wall Street. Con esta disposición se obligaba a las empresas a controlar la procedencia de los minerales (tungsteno, wolframio y tantalio) utilizados para la fabricación de aparatos electrónicos (MartinOrtega, pp. 75-95), cuyo origen proviniera de los territorios en conflictos de la región de los Grandes Lagos, en especial de la República Democrática del Congo. Asimismo, se publicaba la sección 1504 donde en un ejercicio de transparencia sin precedentes se exigía a las empresas energéticas norteamericanas a que publicaran los pagos efectuados a gobiernos que autorizaban dichas extracciones. Lamentablemente la presidencia de Trump y el nombramiento de un alto ejecutivo de la petrolera Exxon Mobile como Secretario de Estado, acabaron por derogar estas disposiciones en una votación de 24 de mayo de 2018, que incluso contó con el apoyo de una minoría de senadores demócratas. Veremos si la nueva administración Biden rescata estas leyes de la administración antecesora demócrata de Obama. Con todo en los Estados Unidos sigue vigente la California Transparency in Supply Chains Act de 2010, que obliga a remitir al Fiscal General del Estado las declaraciones publicadas por las empresas respecto a este tipo de situaciones, el cual puede tomar todo tipo de medidas incardinadas bajo el principio de rendición de cuentas.

Todas estas loables iniciativas legislativas a nivel nacional suponen un considerable avance, aunque queda por analizar en un futuro próximo su eficaz implementación y las consecuencias judiciales en caso de grave incumplimiento. Con todo, debe permanecerse alerta ya que resulta inquietantemente contradictorio que desde estos países se proceda al impulso normativo interno y al mismo tiempo se combata de forma radical y vehemente el proyecto de tratado internacional. Si el propósito común es idéntico, y un mecanismo complementa y refuerza el otro en esta lucha contra la impunidad ante los graves abusos de las empresas

transnacionales, ¿cómo explicar el airado rechazo a unas obligaciones internacionales cuya finalidad coincide con los proyectos normativos nacionales? ¿Por qué ese temor gubernamental al Alien Tort Statute, cuando de forma inminente de nuevo el Tribunal Supremo de los Estados Unidos debe decidir definitivamente en el caso Jerner vs. Arab Bank PLC., el devenir de la exigencia de responsabilidades civiles a las corporaciones (Bernaz, 2017)?¿A qué obedece la posición beligerante, no ya sólo de los Estados Unidos, sino de la Unión Europea, al proyecto de tratado tanto en el Grupo Intergubernamental de Trabajo, como en las deliberaciones y votaciones en el Consejo de Derechos Humanos (Artens \& Seitz, 2016)? Sin ningún género de dudas la respuesta a la retórica pregunta apunta a la alargada sombra de los lobbies corporativos instalados en Washington y Bruselas (Esteve Moltó, 2015, pp. 89-100).

\section{Conclusiones. La necesidad de rescatar el legado de Núremberg}

En la actualidad la mayoría de los abusos ambientales y de derechos humanos de las empresas transnacionales continúan sin tener atribuida una responsabilidad. Aparecen avances a nivel nacional pero con un carácter limitado. Mientras en el plano internacional contrasta la proliferación de directrices, con los malogrados proyectos hasta la fecha de normas vinculantes. Siendo así, los hechos muestran que los códigos de conducta voluntarios y autorregulatorios no 
han sido, ni serán capaces de frenar el avance de la impunidad.

Es por ello, por lo que se requiere de medidas más audaces, y en este sentido la sociedad civil presiona a través del mencionado Grupo Intergubernamental de Trabajo en Naciones Unidas para que el control sobre estos actores no estatales sea una realidad a través de un tratado internacional. Incluso en esta línea se ha venido proponiendo la creación de un tribunal penal internacional especial para las empresas transnacionales (Rigaux, 2002, pp. 128-142). Pero claro está, en esta búsqueda de responsabilidades penales (Kaeb, 2013, pp. 43-77), (Kyriakakis, sf., pp. 221-240), (Magraw, 2009, pp. 458-497) y (Vilt, H. Van Der, 2013, pp. 43-77), para los casos de las más graves violaciones empresariales, no sólo está en debate una cuestión aislada relativa a esta laguna existente y persistente en el derecho internacional. El dar debida respuesta a este gran agujero en la justicia obliga a enfrentar retos que parecen insalvables, como el de si es posible humanizar la globalización económica (Deva, 2012).

En definitiva, el curso del proceso de humanización del derecho internacional ${ }^{29}$ puede verse truncado y el ser humano no podrá desplegar todas sus plenas facultades como sujeto pleno de derecho internacional hasta que estas empresas transnacionales no tengan atribuidas internacionalmente unas obligaciones claras, precisas y exigibles ante una jurisdicción accesible a las víctimas. Es más, las aún no surgidas obligaciones destinadas a estos actores no estatales debieran ya emerger, y no únicamente por cubrir una laguna jurídica en el marco regulatorio internacional apelando a una conciencia jurídica universal. Incluso desde

29. En este contexto son fuente de inspiración los escritos y los fallos suscritos por el juez brasileño Cançado Trindade en los que de forma reiterada apela al espíritu del derecho de gentes. Por citar alguna de sus obras, cfr. Cançado Trindade, A. A., "The emancipation of the individual from his own State- The historical recovery of the human person as subject of the law of nations", en Human rights, democracy and the Rule of Law-Liber Amicorum Luzius Wildhaber (eds. S. Breitenmoser et alii), Dike/Nomos, Zürich/Baden-Baden, 2007, pp. 151171. una perspectiva meramente economicista son evidentes también sus ventajas con el objeto de evitar indeseadas competencias desleales. Y todo ello sin mencionar las mas elementales razones éticas, e incluso de supervivencia del propio planeta en el que los efectos del cambio climático resultan impredecibles, mientras las irrefrenables ansias extractivistas de las corporaciones no pueden ser atajadas por los Estados. Únicamente el solidario compromiso de la sociedad civil puede revertir este proceso suicida, cuya insostenible globalización parece haber secuestrado por parte de las corporaciones el contrato social universal; y mientras tanto los Estados, ya sean los occidentales capitalistas o economías socialistas de mercado, como China, quedan supeditados a los grandes intereses corporativos y a los grandes fondos de inversión, ignorando todos ellos, los valores que de manera hipócrita predican, tanto unos como otros.

En todo caso, el exigir responsabilidades internacionales a las corporaciones, no es una cuestión novedosa propia de un activismo radical de grupos de derechos humanos, a la que se quieren sumar algunos países del Sur, y que pretenden alterar e incluso dinamitar el derecho internacional al convertir a las personas jurídicas en sujetos de derecho en la esfera internacional. Esta exigencia de responsabilidades no es más que la continuación del nuevo orden internacional que se estableció tras la II Guerra Mundial. Estamos ante la continuidad del legado de Núremberg, y en cuyos juicios llegaron a la manifiesta evidencia que, a la hora de poner fin a la impunidad, no era suficiente con atribuir responsabilidades penales a los individuos. Siendo así, si el derecho penal internacional entre sus objetivos consta el sentar las bases de una paz duradera, y necesariamente para poder deshacer la madeja de las causas de los conflictos, de forma inevitable habrá que desenmarañar la compleja red de intereses económicos.

Desde hace décadas resulta tan manifiesto que los perpetradores de los crímenes de guerra pueden ser tanto individuos, como personas jurídicas, que 
negar dicha evidencia resulta insultante para las víctimas. Por ejemplo, en Núremberg fueron condenados dos empresarios de la empresa Zyklon B, que suministraban los gases químicos utilizados en los campos de exterminio. Décadas después el empresario holandés Van Anraat fue condenado por los tribunales nacionales por la venta de agentes químicos al gobierno de Iraq para utilizarlos contra la población kurda. De la misma forma la mera adquisición empresarial de una empresa alemana de una compañía francesa confiscada por el III Reich fue considerado un crimen de guerra. Obviamente el pillaje debe tener una conexión con el conflicto, pero como en el caso sirio comentado, las empresas que operan en una zona de conflicto pueden tener esos nexos de conexión, y la jurisprudencia no requiere de una participación o política directa en las hostilidades.

Si bien es cierto que la jurisprudencia de Núremberg condenó únicamente a personas físicas y no jurídicas, lo relevante es que apuntaron a la complicidad criminal de la industria alemana en el holocausto. Esto es, los crímenes internacionales podían ser cometidos por una amplia "organización criminal"; término "empleado en los juicios de Núremberg para tratar la criminalidad masiva, organizada, voluntaria e intencionada (...)", y cuya interpretación extensiva puede que "alcance a personas jurídicas que tiene como objetivo obtener beneficios privados" y que debe devenir en una ampliación de la competencia rationae personae de la Corte Penal Internacional" (Arenal, 2020, pp. 13-41).

En esta misma línea el Tribunal penal para Ruanda, también condenó a los agentes comerciales de una radio local por incitar a la comisión del genocidio y crímenes de guerra y el Tribunal Penal para la Antigua Yugoslavia en el veredicto de la apelación del caso Tadic no dudó en acudir a la figura de la empresa criminal conjunta. Con posterioridad, el Tribunal Especial para Sierra Leona siguió contemplando el pillaje de recursos naturales como crímenes de guerra. Es por ello que las Convenciones de Ginebra de 1949 prohibieron el pillaje de recursos, tal y como ya contemplaba el art. 6.b del Estatuto de Núremberg, que por cierto, en cierta medida ya estaba contemplado en el DIH desde la normativa de La Haya de 1907 que prohibía de una población o lugar" en el transcurso de la guerra. Así pues, nos encontramos ante una norma y costumbre internacional, aplicable tanto a conflicto armados internacionales, como internos, tal y como dispone el art. 4.2.g del Protocolo Adicional II.

Décadas después el Tribunal Penal Internacional sigue con esta misma laguna, a pesar de la tentativa de avance que supuso la primera sentencia del caso Bemba que ha sido finalmente revocada. Y es por ello, que las críticas a este órgano de practicar un neocolonialismo jurídico en África, resulta difícil de combatir. Si fuera de otro modo, de forma decidida en las distintas investigaciones abiertas se hubiera podido tirar del hilo de la complicidad criminal, y junto a militares y mandatarios africanos irían apareciendo las actuaciones de distintas transnacionales o sus filiales que con avidez acaparan tierras y explotan recursos naturales, valiéndose de la violencia. Es por ello, que el Protocolo a la Carta Africana de Derechos Humanos, en su sección penal habilita la persecución de violaciones cometidas por empresas africanas (filiales) o extranjeras que operan en África, e incluye el crimen explotación ilegal de recursos naturales (art. 28 $8^{a}$ y 46C). Es más, se prevé que para activar la competencia de este tribunal regional bastaría hacer constar que la violación afecte a un interés de un Estado Parte. Lamentablemente el Protocolo de Malabo aún no ha podido reunir las quince ratificaciones que exige su artículo 11 para poder entrar en vigor. De igual manera esa misma carga de profundidad pretende ostentar el Tribunal Penal Extraordinario para Liberia que pretende enjuiciar también a personas jurídicas.

Ahora bien, un cambio de paradigma en las leyes y tribunales nacionales es y debe ser posible. El caso Lafarge y Amesys en Francia, el caso Rabobank ante tribunales holandeses, los asuntos de la minera Argor Hereaus o Nestlé ante la justicia suiza, el de la petrolera 
Unocal ante tribunales californianos y la exigencia judicial de responsabilidades civiles a las corporaciones ante la justicia estadounidense, con sus avances y retrocesos (Marullo \& Zamora, 2016), (Zamora Cabot, 2005, pp.1381-1394), (Pigrau Solé, 2009, pp.
113-130), han ido abriendo las primeras vías de lucha contra la impunidad en este ámbito. La lucha contra la impunidad parece titánica, el derecho internacional en este ámbito parece herido de muerte, pero sea como fuere, la resistencia continúa.

\section{REFERENCIAS BIBLIOGRÁFICAS}

» Adeola, R. (2017). "Why the African Union must press ahead with a business and human rights policy", The conversation, 8 mayo, en http://theconversation. com/why-the-african-union-must-press-ahead-with-a-business-and-humanrights-policy-75712.

» Arenal Lora, L. (2020). "La comisión de crímenes contra la humanidad por empresas transnacionales y la ampliación de la competencia rationae personae de la Corte Penal Internacional", Deusto Journal of Human Rights, $n^{\circ}$ 6, pp. 13-41.

» Artens, J. \& Seitz, K. (2016). The Struggle for a UN Treaty. Towards global regulation on human rights and business. Global Policy Forum/Rosa Luxemburg Stiftung, Berlin, Bonn, New York, disponible en https://www.globalpolicy.org/images/ pdfs/UN_Treaty_online.pdf.

ASEAN. (2017). ASEAN conduct training on the linkages between business activities and human rights", Bangkok, 21 noviembre. Recuperado de: http://aichr. org/press-release/asean-conduct-training-on-the-linkages-between-businessactivities-and-human-rights/.

» Bakan, Joel. (2004). The Corporation: The Pathological Pursuit of Profit and Power. New York: Free Press.

» Baxi, U., (2010). "Writing about impunity and environment: the 'silver jubilee' of the Bhopal catastrophe", Journal of Human Rights and Environment, vol. 1, $n^{\underline{o}}$ 1, pp. 23-44, p. 39, disponible en http://www.elgaronline.com/view/journals/ jhre/1-1/jhre.2010.01.02.xml.

» Burma Campaign, UK. (2008). Dirty list, Londres. Recuperado de www.burmacampaign.org.uk/dirty_list/dirty_list_details.html.

» Bernaz, N. (2017) The Moment of Truth Has Come - US Supreme Court to Decide whether the Alien Tort Statute "Categorically Forecloses Corporate Liability", Rights as Usual, 3 abril, recuperado de: http://rightsasusual.com/?p=1140.

» Business and Human Rights Resource Centre. (2018). "Canada creates independent Ombudsperson \& multi-stakeholder advisory body to strengthen responsible business conduct abroad", 25 enero. Recuperado de: https://business-humanrights.org/en/canada-creates-independent-ombudsperson-multi-stakeholderadvisory-body-to-strengthen-responsible-business-conduct-abroad.

» Dale, John. (2011). Free Burma: Transnational legal action and corporate accountability, University of Minessota Press.

» Cervera Vallterra, M. (2016). "La fragilidad de la República Democrática del Congo: Problemas y soluciones a la posesión de recursos minerales", Revista electrónica de estudios internacionales (REEI), №. 31.

Debouzy, A. (2017). “Censure partielle, mais sévère, par le Conseil Constitu- 
tionnel, de la loi relative au devoir de vigilance des sociétés mères et des entreprises donneuses d'ordre", Lexology, 24 marzo.

» Deva, S. (2012). Regulating Corporate Human Rights Violations: Humanizing Business, Routledge, London/New York,

» Díaz Barrado, C.M. (2016). "Los objetivos de desarrollo sostenible: un principio de naturaleza incierta y varias dimensiones fragmentadas", Anuario Español de Derecho Internacional, vol. 32, pp. 9-48.

» Diller, J. (2000). "Social conduct in transnational enterprise operations: the role of the International Labour Organization", en Blanpain (ed.), Multinational Entreprises and the Social Challenges of the XXIst Century. Bulletin of Comparative Labour Relations, 37. Kluwer Law International, The Hague, London, Boston.

¿ ECCHR. (2016). Case report. Lafarge in Syria: accusations of complicity in war crimes and crimes against humanity, noviembre.

» Esteve Moltó, J.E. (2015). “La 'gobernanza' y control de las corporaciones transnacionales sobre los valores, objetivos y principios de la Unión Europea y sus ciudadanos", en García Sáez, J.A. \& Vañó Vicedo, R., Educar la mirada: documentales para una enseñanza crítica de los Derechos Humanos, Tirant Lo Blanch, Valencia, pp. 89-100. (978-84-9086-634-4).

» Jiménez Sánchez, J. J. (2006). “Marcos de las relaciones laborales en las empresas multinacionales: las directrices de la OCDE", en Empresas multinacionales: su incidencia en las relaciones sociales, Cuadernos de Derecho Judicial, Consejo General del Poder Judicial, Madrid.

" Grear, A. \& Weston, B.H. (2015). "The Betrayal of Human Rights and the Urgency of Universal Corporate Accountability: Reflections on a Post-Kiobel Lawscape", Human Rights Law Review, vol. 15, pp. 21-44.

» Fikentscher, W. (1982). "United Nations code of conduct: new paths in International Law", American Journal of Comparative Law, 30, 4, pp. 577-604.

» Hillemanns, C. (2003). "UN Norms on the responsibilities of transnational corporations and other business enterprises with regard to human rights", German Law Journal 4, pp. 1065-1080.

» Jägers, Nicola M.C.P. (2002). Corporate Human Rights obligations: in search of accountability, ed. Intersentia, School of Human Rights Research Series, volume 17, Antwerpen, pp. 124-128.

» Kaeb, C. (2016). "The Shifting Sands of Corporate Liability under International Criminal Law", The George Washington International Law Review Vol 49, pp. 351403.

» Kenney, C. (2009). "Disaster in the Amazon: Dodging "Boomerang Suits" Transnational Human Rights Litigation, California Law Review 97, pp. 857 y ss.

» Kimerling, J. (2006). “Transnational Operations, Bi-National Injustice: Chevron Texaco and Indigenous Huaorani and Kichwa in the Amazon Rainforest in Ecuador", American Indian Law Review 31, pp. 445 y ss.

» Kyriakakis, J. (s,f,) “Corporations before International Criminal Courts: Implications for the International Criminal Justice Project", Leiden Journal of International Law, 30 (1), pp. 221-240.

» Lutz, R.E. (1988). "The export of danger: a view from the developed world", 
New York University Journal of International Law and Politics 20, pp. 629-677.

$»$ Magraw, K. (2009). "Universally Liable? Corporate-Complicity Under the Principle of Universal Jurisdiction", Minnessota Journal of International Law, vol. 18, pp. 458-497.

» Manirabona, A. M. (2011). "L'affaire Trafigura: vers la répression de graves atteintes environnementales en tant que crimes contre l'humanité", Revue de droit internationale et de droit comparé, Vol. 88, №. 4, pp. 535-576.

» Márquez Carrasco, M.C. (2017). "Elementos claves de la implementación de los principios rectores de las Naciones Unidas sobre empresas y derechos humanos en la Unión Europea" en Márquez Carrasco y Vivas Tesón (coord..), La implementación de los principios rectores de las Naciones Unidas sobre empresas y derechos humanos por la Unión Europea y sus estados miembros, Thomson Reuters-Aranzadi : Ministerio de Economía y Competitividad, pp. 21-47.

» Márquez Carrasco, M.C. (2015). "La implementación de los principios rectores de las Naciones Unidas sobre empresas y derechos humanos por medio de los planes nacionales de acción", Revista de responsabilidad social de la empresa, no 20 , pp. 55-87.

» Martin-Ortega, O. (2013). "La diligencia debida de las empresas en materia de derechos humanos: un nuevo estándar para una nueva responsabilidad", Papeles El Tiempo de los Derechos, pp. 15 y ss.

» Martin-Ortega, O. (s.f.) "Public procurement as a tool for the protection and promotion of Human Rights: a study of collaboration, due diligence and leverage in the electronics industry", Business and Human Rights Journal, Vol. 3, issue 1, pp. 75-95.

» Marullo, M.C. \& Zamora Cabot, F.J. (2016). "Transnational Human Rights litigations. Kiobel's touch and concern: a test under construction", Papeles El Tiempo de los derechos $n^{\circ}$ 1, disponible en https://redtiempodelosderechos.files.wordpress. com/2015/01/wp-1-16.pdf.

» Moon, G. (2013). “Trading in Good Faith? Importing States' Economic Human Rights Obligations into the WTO's Doha Round Negotiations", Human Rights Law Review, pp. 1-41.

" Nseeuw, W. (2013). "The Rush for Land in Africa: Resource Grabbing or Green Revolution?, South African Journal of International Affairs, vol. 20, pp. 159-177.

» OCDE. (2013). Líneas Directrices de la OCDE para Empresas Multinacionales, en http://dx.doi.org/10.1787/9789264202436-es

$» \quad$ OCDE. (2017). Due Diligence Guidance for Responsible Supply Chains in the Garment and Footwear Sector, en https://mneguidelines.oecd.org/OECD-Due-Diligence-Guidance-Garment-Footwear.pdf.

» Organización Internacional del Trabajo -OIT-. (2001). Declaración Tripartita de Principios sobre las Empresas Multinacionales y la Política Social, Oficina Internacional del Trabajo, recuperado de: http://www.ilo.org/wcmsp5/groups/public/ed_emp/documents/publication/wcms_124924.pdf.

» Patel, K. \& N. Nyembe. (2017). "Hacia un tratado vinculante”, Por la paz $n^{\circ}$ 30, Empresas y Derechos Humanos, febrero.

» Pigrau Solé, A. (2014). “The Texaco-Chevron case in Ecuador: law and justice in the age of globalization", Revista Catalana de Dret Ambiental, vol. V, no 1, pp. 1-43, disponible en http://www.rcda.cat/index. php/rcda/article/view/491. 
» Pigrau Solé, A. (2009). "La Responsabilidad Civil de las Empresas Transnacionales a Través de la Alien Tort Claims Act por su Participación en Violaciones de Derechos Humanos", Revista Española de Desarrollo y Cooperación, nํㅡㄹ 25, pp. 113130.

» Rigaux, F. (2002). "Toward an international criminal tribunal to adjudicate upon corporate wrongs", Transnational Associations 53, 2, pp. 128-142. Del mismo autor, “¿Un tribunal (penal) internacional para las sociedades transnacionales?", Informe del seminario, Les activités transnationales et la nécessité de leur encadrement juridique, Centre Europe-Tiers Monde y Asociación Americana de Juristas, Cetim /AAJ, Geneve, 2001 en http://www.cetim.ch/es/documents/ esppart1.pdf.

» Sánchez Patrón, J.M: "Responsabilidad ambiental y derechos humanos: Los deberes de las empresas en el Derecho Internacional", REEI, nº 32, diciembre 2016.

» Stiglitz, Joseph E (2007). "Multinational Corporations: Balancing Rights and Responsibilities." Proceedings of the American Society of International Law 101, pp. 3-60.

» Thomson Reuters Foundation. (2018). "UK: Traffickers of Vietnamese women for UK nail salon serve prison time; case is the first successful prosecution under the Modern Slavery Act", 3 de enero. Recuperado de: https://business-humanrights.org/en/uk-traffickers-who-enslaved-vietnamese-girls-for-nail-salon-jailed-under-under-the-modern-slavery-act\#c166563

» UN Working Group on Business and Human Rights, Guidance on National Action Plans on Business and Human Rights, Ginebra, noviembre 2016, disponible en http://ohchr.org/Documents/Issues/Business/UNWG_NAPGuidance.pdf.

» Vilt, H. Van Der: "Corporate Criminal Responsibility for International Crimes: Exploring the Possibilities", Chinese Journal of International Law, vol. 12, 2013, pp. 43-77.

» Wallace, R.M.M. y Martin-Ortega, O.: "The UN Norms: a first step to universal regulation of transnational corporations' responsibilities for human rights?", Dublin University Law Journal 26, pp. 304-319.

» Weilert, Katarina: "Taming the untamable? Transnational corporations in United Nations law and practice", Max Planck Yearbook of United Nations Law, vol. 14, 2010, pp. 445-506, en concreto ver, pp. 491-504.

»Weissbrodt, D. y Kruger, M. (2003). "Norms on the responsabilities of transnacional corporations and other business enterprises with regard to human rights", American Journal of International Law 97, 4, pp. 901-922.

»Wells, C. y Elias, J. (2005). "Catching the consciente of the king: corporate players on the international stage", en Alston, P. (ed.): Non-state actors and human rights, Oxford University Press, Oxford, pp. 141-175, p. 148.

» Zamora Cabot, F.J. (2016). "Kiobel vs. Royal Dutch Corp. y los litigios transnacionales sobre derechos humanos", Derechos humanos y políticas públicas europeas, coord. por Daniel Innerarity Grau, Ignacio Aymerich Ojea, Paidós, pp. 149-166.

» Zamora Cabot, F.J. (2013). "Acaparamiento de Tierras (Land Grabbing) y Empresas Multinacionales: El Caso Mubende-Neumann", en Papeles El Tiempo de los Derechos.

» Zamora Cabot, F.J. (2012). "La Responsabilidad de las Empresas Multinacionales por Violaciones de los Derechos Humanos: Práctica Reciente", Anuario de los 
Cursos de Derechos Humanos de Donostia- San Sebastián, vol. 12, pp. 194-207.

» Zamora Cabot, F. J. (2005). "Una Luz en el Corazón de las Tinieblas: El Alien Tort Claims Act of 1789 (ATCA) de los Estados Unidos" en Soberanía del Estado y Derecho Internacional, Homenaje al Prof. J.A. Carrillo Salcedo, Universidades de Córdoba, Sevilla y Málaga, Sevilla, pp.1381-1394.

» Zolin, M. B., y Braggion, M. (s.f.). "Land Grabbing, Food Security and Energy Security in Asia: The Cases of China and India", en http://www.wbiworldconpro. com/uploads/malaysia-conference-2013/economics/220-Maris.pdf 